\title{
Zooplankton Foraging induces Chaos in Dynamic Green Ocean Models
}

\author{
$\underline{\text { R.A. Cropp }}^{\text {a }}$, I.M. Moroz ${ }^{\text {b }}$ and J. Norbury ${ }^{b}$ \\ ${ }^{a}$ Griffith School of Environment, Griffith University, Gold Coast, Australia \\ ${ }^{b}$ Mathematical Institute, University of Oxford, Andrew Wiles Building, ROQ, Woodstock Road, Oxford. \\ Email: $\underline{\text { r.cropp@griffith.edu.au }}$
}

\begin{abstract}
The exchange of important greenhouse gases between the ocean and atmosphere is influenced by the dynamics of near-surface plankton ecosystems. Marine plankton ecosystems are modified by climate change creating a feedback mechanism that could have significant implications for predicting future climates, for example, the collapse or extinction of a plankton population may push the climate system across a tipping point. Dynamic green ocean models (DGOMs) are currently being developed for inclusion in climate models to predict the future state of the climate. These models are often complicated, commonly with 5-10 competing phytoplankton and several omnivorous zooplankton (Le Quéré et al., 2005). Complicated dynamics including chaos are readily found even in unforced forms of the relatively simple nonlinear plankton ecosystem ordinary differential equation models that underpin DGOMs (Cropp et al., 2014). The appropriate complexity of the DGOMs is an ongoing issue, with models tending to become more complex, and perhaps an increasing propensity for chaos (Fussmann and Heber, 2002).

The complexity of DGOMs means that most attempts to confer them with "desirable" properties proceed by numerical experimentation and/or model inter-comparison projects such as the MARine Ecosystem Model Inter-comparison Project (MAREMIP, Sailley et al., 2013). Some recent investigations into DGOMs have considered the role of zooplankton predation functional forms in determining model properties (for example, Anderson et al., 2010; Visser and Fiksen, 2013; Vallina et al., 2014). The functional forms considered in these experiments are generally based on the classic Holling Type II or III forms (Holling, 1959) with modifications to represent zooplankton strategies such as the specialised or generalised feeding strategies described by Koen-Alonso (2007) and prey switching (Gentleman et al., 2003). However, little consensus has been reached on the most useful form of grazing function for plankton systems that underpin both green ocean models and fisheries ecologies (Le Quéré et al., 2005).
\end{abstract}

We consider a relatively simple (three-population) DGOM of two phytoplankton and a zooplankton where the interacting plankton populations compete for a single limiting nutrient. We find chaotic dynamics are possible in this low trophic order ecological model with a specialist foraging strategy as we vary the zooplankton mortality. This suggests that chaotic dynamics might be ubiquitous in the more complex models, but this is rarely observed in DGOM simulations. The physical equations of DGOMs are well understood and are constrained by conservation principles, but the ecological equations are not as well understood, and are often constructed without explicit consideration of conserved quantities as closed model domains are considered unrealistic by some ecologists (Loreau, 2010, p 16). The work we present here utilizes a theoretical framework constructed on the fundamental principles of conservation of mass, finite resources and explicit resource limitations to growth. Our results, when considered in the context of the paucity of the empirical and theoretical bases upon which DGOMs are constructed, raises the interesting question of whether DGOMs would represent reality better if they include or exclude chaotic dynamics.

Our analysis of this simple, but representative, plankton system suggests that apparently innocuous choices of grazing terms, varying from indiscriminate to discriminate types which do not appear significantly different, and which may be equivalent up to observational/experimental accuracy, can predetermine the emergent properties of the systems. We observe that the indiscriminate grazer appears to have more reliable and steadier shares of the ecosystem biomass in contrast to the discriminate grazer's very strongly fluctuating biomass share. Indiscriminate grazing functions for zooplankton are commonly used in the current generation of GCMs, where the emphasis is to maintain biodiversity and to represent the dynamics of large groups of plankton functional types (PFTs). However, future generations of GCMs may wish to resolve more detailed dynamics, such as bloom succession, and we suggest that for these models introducing discriminate grazing functions for the marginal populations may be more appropriate.

Keywords: Chaos, plankton model, CN framework, zooplankton foraging, zooplankton mortality 


\section{INTRODUCTION}

The choice of the functional form to represent interactions between populations in ecosystems is an ongoing point of contention among ecologists. The theoretical bases for many of the simple two-population interaction terms were set out early [i.e. (Lotka, 1925; Volterra, 1926; Holling, 1959; Ivlev, 1961)] but there remains little theoretical or empirical evidence to justify a choice of one form over another. A number of studies have suggested that even simple functional forms may have important influences on properties of the systems. For example, Steele and Henderson (1992) observed that the functional form of zooplankton mortality, often a closure term in plankton models, affected the dynamical stability of simple models, with linear mortality apparently destabilising the system. However, it often turns out that things are more complicated than they first appear, and it now seems that there is no simple relationship between stability and the form of the closure term (Edwards and Brindley, 1996, 1999; Edwards and Yool, 2000).

The Conservative Normal (CN) framework underpins the theoretical basis for our modeling approach (Cropp and Norbury, 2012a, b, 2013; Cropp et al., 2014; Cropp and Norbury, 2015). It identifies clearly models that are ecologically unrealistic and allows some emergent properties to be predetermined. For example, Cropp and Norbury (2012b) showed that for a general class of models it was possible to ensure coexistence in a model of any complexity by choosing functional forms for loss terms that vanished as the population vanished. The simple form of the boundary eigenvalues in $\mathrm{CN}$ systems, which are all known analytically, may be used to provide pre-determined properties such as community structure. The choices made for the functional forms of population interactions can be important pre-determinants of system properties. Here, we identify a priori some zooplankton properties that have not been considered in previous analyses of grazing in DGOMs, and have strongly related, but very different, dynamical behaviours.

The $\mathrm{CN}$ framework allows us to differentiate between normal and obligate grazing, where we define normal grazing to mean that the zooplankton population can survive on any one of its prey independently. We define obligate grazing to mean that the zooplankton population is dependent on a specific prey being present in order for it to survive - although it also consumes and grows on its other prey, it cannot survive if its obligate prey is absent. We also consider whether the zooplankton functions as an indiscriminate or discriminate grazer, analogous to the generalists and specialists defined by Koen-Alonso (2007). Rather than consider these processes in the complicated domain of a "realistic" fully coupled global biophysical model, we take the simpler "caricature model" approach advocated by many investigators that is more amenable to analysis and hence can often provide unequivocal, though constrained, answers. In this paper we fix on a linear mortality term for zooplankton and examine the dramatic differences in system dynamics that appear after quite mild variations in the zooplankton foraging strategy.

\section{ZOOPLANKTON FORAGING STRATEGIES}

Zooplankton are known to move up and down actively through the water column to optimise their feeding strategy, with recent observations revealing that Antarctic krill even dive to abyssal depths to feed (Clarke and Tyler, 2008). This suggests that more sophisticated feeding functions than the standard indiscriminate multi-prey feeding functions (Gentleman et al., 2003) should be used to model zooplankton grazing. Indiscriminate grazing functions assume that foraging zooplankton will ingest any prey that they encounter during a generic foraging expedition. We also consider two feeding relations: obligate grazers that cannot survive if their obligate prey is absent; and facultative (or normal) grazers that can survive on either of their prey independently.

\subsection{Discriminate/indiscriminate Grazing}

We differentiate discriminate grazers as zooplankton that actively divide the time they spend foraging for prey between two states, for example, shallow and deep so that they optimise their feeding rates on each prey. We make two assumptions regarding discriminate grazing: that discriminate grazing could not persist in a population unless it provides some advantage to the grazer, and that a discriminate grazer would adjust the proportions of time devoted to searching for each prey according to prey abundance. We then expect a discriminate grazer to have a better capture rate for a given prey and that in the absence of alternative prey, indiscriminate and discriminate grazing strategies are equivalent. We consider a grazer $Z$ feeding on two prey populations $\left(P_{1}\right.$ and $\left.P_{2}\right)$ and use the discriminate (sometimes referred to as specialist) feeding function (where $\phi_{i}$ represents the fraction of foraging time allocated to each prey):

$$
G_{D}=\phi_{1} \frac{\hat{\varphi}_{1} P_{1}}{1+\hat{\varepsilon}_{1} P_{1}}+\phi_{2} \frac{\hat{\varphi}_{2} P_{2}}{1+\hat{\varepsilon}_{2} P_{2}},
$$


and the indiscriminate (or generalist) feeding function:

$$
G_{I}=\frac{\varphi_{1} P_{1}}{1+\varepsilon_{1} P_{1}+\varepsilon_{2} P_{2}}+\frac{\varphi_{2} P_{2}}{1+\varepsilon_{1} P_{1}+\varepsilon_{2} P_{2}},
$$

where the parameters are defined in section 2.3. To facilitate an equitable comparison between grazing functions, free of the influence of artefacts from different parameter values, we require that discriminate and indiscriminate grazers perform equally well when only one prey exists that is:

$$
\frac{\varphi_{i} P_{i}}{1+\varepsilon_{i} P_{i}}=G_{I, i}=G_{D, i}=\phi_{i} \frac{\hat{\varphi}_{i} P_{i}}{1+\hat{\varepsilon}_{i} P_{i}}, \quad i=1,2 .
$$

This assumption dictates that $\varphi_{i}=\phi_{i} \hat{\varphi}_{i}$, implicitly meaning that the discriminate grazer optimises its foraging strategy according to the relative abundance of the prey populations. To enable comparison of the grazing functional forms on equal terms we reduce the number of confounding factors and set $\varepsilon_{i}=\hat{\varepsilon}_{i}$. This assumption makes a direct ecological interpretation of our results less intuitive, but we take the view that the effect of the functional forms is more important than the effect of these parameter values, and the relatively straight-forward examination of the influence of parameter values within a particular grazing strategy is easily undertaken by those with particular interest in this aspect.

We combine the two grazing forms (1) and (2) as in equation (4) to enable us to use a simple computational switch to transition between discriminate and indiscriminate grazing types using a single parameter $(\rho)$. This allows us to change the functional form of grazing within the same computer model and minimises the risk of coding errors influencing our conclusions. To simplify our computational study, we assume that the prey have identical properties but that the zooplankton preferentially consumes $P_{1}$ (i.e. $\varphi_{1}>\varphi_{2}$ ).

\subsection{Normal/obligate Grazing}

We use the $\mathrm{CN}$ framework as the theoretical basis for the construction of our model and the determination of the feeding attributes of the zooplankton (Cropp and Norbury, 2015). To define obligate versus normal omnivory, we consider parameter ranges where $Z$ can always survive on $P_{1}$ alone $\left(0<\sigma_{Z}<\sigma_{Z}^{+}\right.$, where $\sigma_{Z}$ is the zooplankton mortality rate and $\sigma_{Z}^{+}$is defined in equation (6)), can survive on $P_{1}$ and/or $P_{2}$ alone ( $0<\sigma_{z}<\sigma_{z}^{-}$, where $\sigma_{z}^{-}$is defined in equation (5)) but can't survive on $P_{2}$ alone when $\sigma_{z}^{-}<\sigma_{z}<\sigma_{z}^{+}$. Here $\sigma_{Z}^{-}$gives the transition from normal grazing to obligate grazing on $P_{1}$ as the grazer mortality rate $\sigma_{Z}$ increases past $\sigma_{Z}^{-}$(remember $Z$ always prefers, or grows better on, $P_{1}$ ).

The $\mathrm{CN}$ maximum resource per capita grazing sign condition for $Z$ provides the parameter constraint:

$$
\frac{\varphi_{1}\left(1-\psi_{1}\right) P_{1}}{1+\varepsilon_{1} P_{1}+\rho \varepsilon_{2} P_{2}}+\frac{\varphi_{2}\left(1-\psi_{2}\right) P_{2}}{1+\rho \varepsilon_{1} P_{1}+\varepsilon_{2} P_{2}}-\sigma_{Z}>0 \text { for } \quad P_{1}+P_{2}=1
$$

We define $Z$ to be a normal grazer, or alternatively a "facultative grazer", if it can survive on either $P_{1}$ or $P_{2}$ independently, that is, we evaluate (4) at the extremities $P_{1}=1$ and $P_{2}=1$ and obtain the constraint:

$$
0<\sigma_{Z}<\min \left\{\frac{\varphi_{1}\left(1-\psi_{1}\right)}{1+\varepsilon_{1}}, \frac{\varphi_{2}\left(1-\psi_{2}\right)}{1+\varepsilon_{2}}\right\} \equiv \frac{\varphi_{2}\left(1-\psi_{2}\right)}{1+\varepsilon_{2}} \equiv \sigma_{Z}^{-} .
$$

This complies with the $\mathrm{CN}$ definition of a "normal" ecology. We also consider the case of an obligate CN ecology, where $Z$ has the obligate property for the parameter range:

$$
\sigma_{Z}^{-}<\sigma_{Z}<\max \left\{\frac{\varphi_{1}\left(1-\psi_{1}\right)}{1+\varepsilon_{1}}, \frac{\varphi_{2}\left(1-\psi_{2}\right)}{1+\varepsilon_{2}}\right\} \equiv \frac{\varphi_{1}\left(1-\psi_{1}\right)}{1+\varepsilon_{1}} \equiv \sigma_{Z}^{+} .
$$

In this case we define $Z$ to be an "obligate grazer", obligate in the sense that it must have $P_{1}$ present in order to survive (remember that we order the prey so that the first term in (6) is the larger). Using the growth and grazing parameter values (13), $Z$ is a normal (facultative) grazer if:

$$
0<\sigma_{Z}<\sigma_{Z}^{-}=0.1708
$$


and is an obligate grazer (it cannot survive in the absence of $P_{1}$ ) if:

$$
\sigma_{Z}^{-}=0.1708<\sigma_{Z}<0.5705=\sigma_{Z}^{+} \text {. }
$$

If $\sigma_{Z}>0.5705$ then $Z$ is not a viable population as it cannot grow, or therefore survive, under any ecologically realistic circumstance. Note that the definition of $Z$ as a normal or obligate grazer is independent of its foraging strategy, so we must examine all four combinations of indiscriminate and discriminate feeding with normal and obligate omnivory.

We note that grazing formulations of the form (1) are sometimes criticised in consumer-resource models that do not have mass conservation because they allow unbounded grazing - as the number of prey populations increases, the total grazing also increases without bound. However, when they are implemented in models where mass is conserved (as is fundamental to the $\mathrm{CN}$ framework) grazing is always bounded. The advantage of discriminate grazing in this case indicates advantages gained by the grazer as a result of using a more sophisticated foraging strategy.

\subsection{The NPPZ Model}

Our approach considers a minimal $N P_{1} P_{2} Z$ model of a single limiting nutrient $(N)$, two phytoplankton populations $\left(P_{1}\right.$ and $P_{2}$ ) and a single zooplankton population $(Z)$ that grazes on both $P_{1}$ and $P_{2}$ (equations (9) -(12)). The zooplankton grazing function includes a parameter $(\rho)$ that allows the functional form to transition smoothly from indiscriminate grazing $(\rho=1)$ to discriminate grazing $(\rho=0)$.

$$
\begin{gathered}
\dot{P}_{1}=P_{1} f_{P_{1}}=P_{1}\left[\frac{\mu_{1} N}{N+\kappa_{1}}-\frac{\varphi_{1} Z}{1+\varepsilon_{1} P_{1}+\rho \varepsilon_{2} P_{2}}-\sigma_{1}\right] \\
\dot{P}_{2}=P_{2} f_{P_{2}}=P_{2}\left[\frac{\mu_{2} N}{N+\kappa_{2}}-\frac{\varphi_{2} Z}{1+\rho \varepsilon_{1} P_{1}+\varepsilon_{2} P_{2}}-\sigma_{2}\right] \\
\dot{Z}=Z f_{Z}=Z\left[\frac{\varphi_{1}\left(1-\psi_{1}\right) P_{1}}{1+\varepsilon_{1} P_{1}+\rho \varepsilon_{2} P_{2}}+\frac{\varphi_{2}\left(1-\psi_{2}\right) P_{2}}{1+\rho \varepsilon_{1} P_{1}+\varepsilon_{2} P_{2}}-\sigma_{Z}\right] \\
\dot{N}=Z\left[\sigma_{Z}+\frac{\varphi_{1} \psi_{1} P_{1}}{1+\varepsilon_{1} P_{1}+\rho \varepsilon_{2} P_{2}}+\frac{\varphi_{2} \psi_{2} P_{2}}{1+\rho \varepsilon_{1} P_{1}+\varepsilon_{2} P_{2}}\right]-P_{1}\left[\frac{\mu_{1} N}{N+\kappa_{1}}-\sigma_{1}\right]-P_{2}\left[\frac{\mu_{2} N}{N+\kappa_{2}}-\sigma_{2}\right]
\end{gathered}
$$

We produce the numerical investigations by integrating equations (9)-(12) with the arbitrary, but realistic, parameter set based on values measured in the field (see (Cropp and Norbury, 2009) for details):

$$
\begin{aligned}
& \mu_{1}=1.00, \quad \kappa_{1}=0.25, \quad \varphi_{1}=6.18, \quad \psi_{1}=0.40, \quad \varepsilon_{1}=5.50, \quad \sigma_{1}=0.00, \quad \sigma_{z}=0.19 . \\
& \mu_{2}=1.15, \quad \kappa_{2}=0.14, \quad \varphi_{2}=1.85, \quad \psi_{2}=0.40, \quad \varepsilon_{2}=5.50, \quad \sigma_{2}=0.26 \text {, }
\end{aligned}
$$

Here, the $\mu_{i}$ are maximum phytoplankton growth rates, the $\kappa_{i}$ are nutrient half-saturation constants, the $\varphi_{i}$ are zooplankton grazing rates, the $\psi_{i}$ are zooplankton assimilation efficiencies, the $\varepsilon_{i}$ are related to the handing times of the prey and the $\sigma_{i}$ represent natural mortality and mortality due to higher predation.

\section{RESULTS}

We consider the properties of the system for continuous variation of $\sigma_{Z}$ for indiscriminate foraging ( $\rho=1$, Fig. 1) and discriminate foraging ( $\rho=0$, Fig. 2). The dynamics of the system with indiscriminate foraging are relatively simple: the system has a unstable equilibrium state comprised of $\left\{P_{2}, Z, N\right\}$ for low values of $\sigma_{Z}$ surrounding which is a stable limit cycle involving periodic blooms of $P_{2}, Z$ and $N$ for $0<\sigma_{Z}<0.11$ after which a stable $P_{2}, Z$ and $N$ steady state exists (Fig 1b-d). This stable coexistence is maintained, with $P_{2}$ rapidly increasing and $Z$ rapidly reducing in response to further increases in $\sigma_{Z}$ until the $P_{1}$ population invades when $\sigma_{Z} \approx 0.17$ (Fig 1a) As $\sigma_{Z}$ increases further, the equilibrium population of $P_{1}$ increases linearly, $P_{2}$ reduces commensurately, and the equilibrium $Z$ population is constant, as is the inorganic 
nutrient $N$ (Fig $1 \mathrm{c}$,d). This continues until $P_{2}$ becomes zero (at $\sigma_{Z} \approx 0.55$ ) after which $P_{1}$ and $Z$ briefly stably coexist until $Z$ goes extinct at $\sigma_{Z}=\sigma_{Z}^{+}=0.5705$.
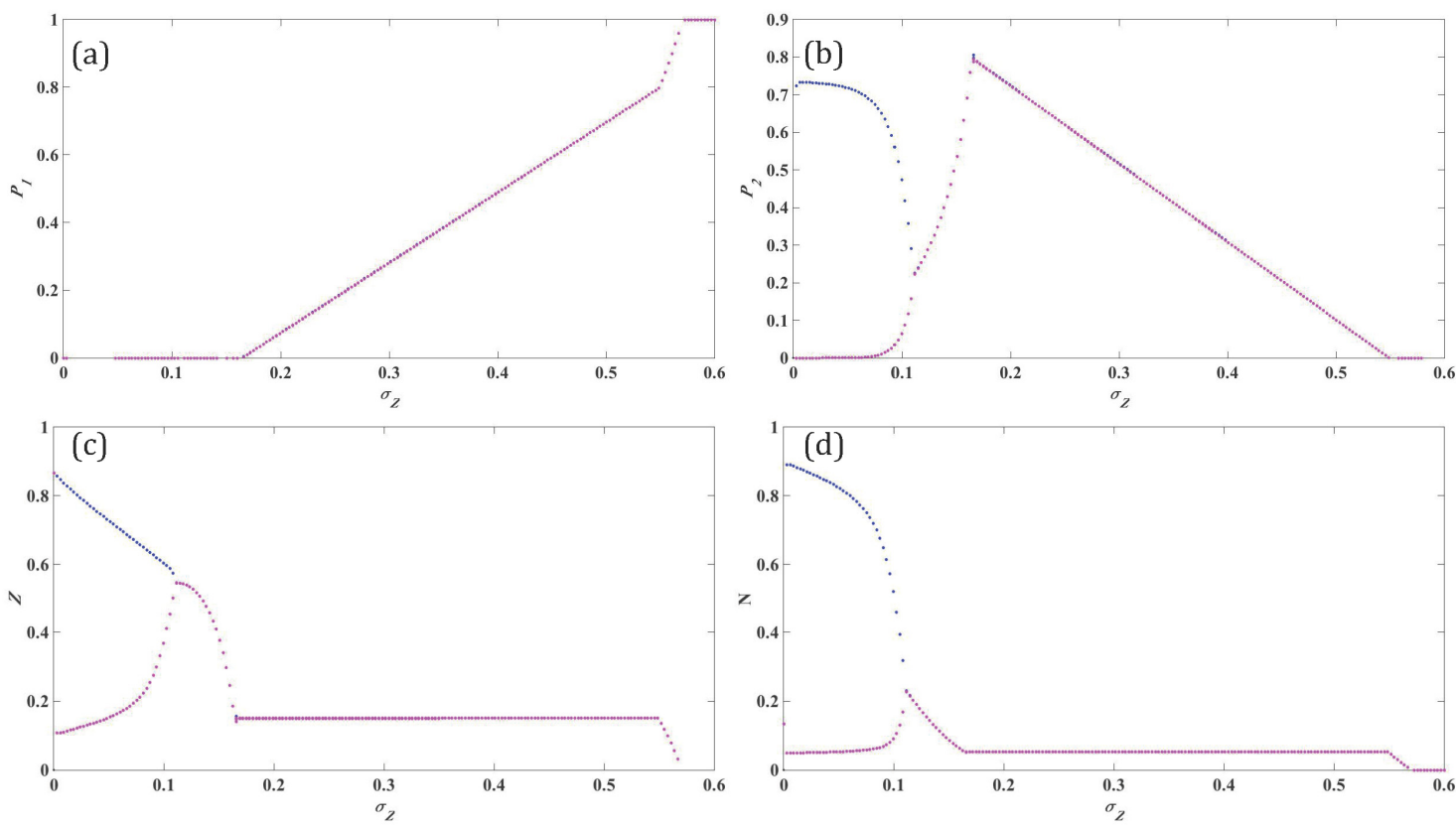

Figure 1. Bifurcation transition diagrams for the indiscriminate forager ( $\rho=1)$ for $P_{1}$ (a), $P_{2}$ (b), $Z$ (c), and $N$ (d) for values of the parameter $0<\sigma_{Z}<0.6$. Local maximums are shown as blue dots and local minimums as red dots.
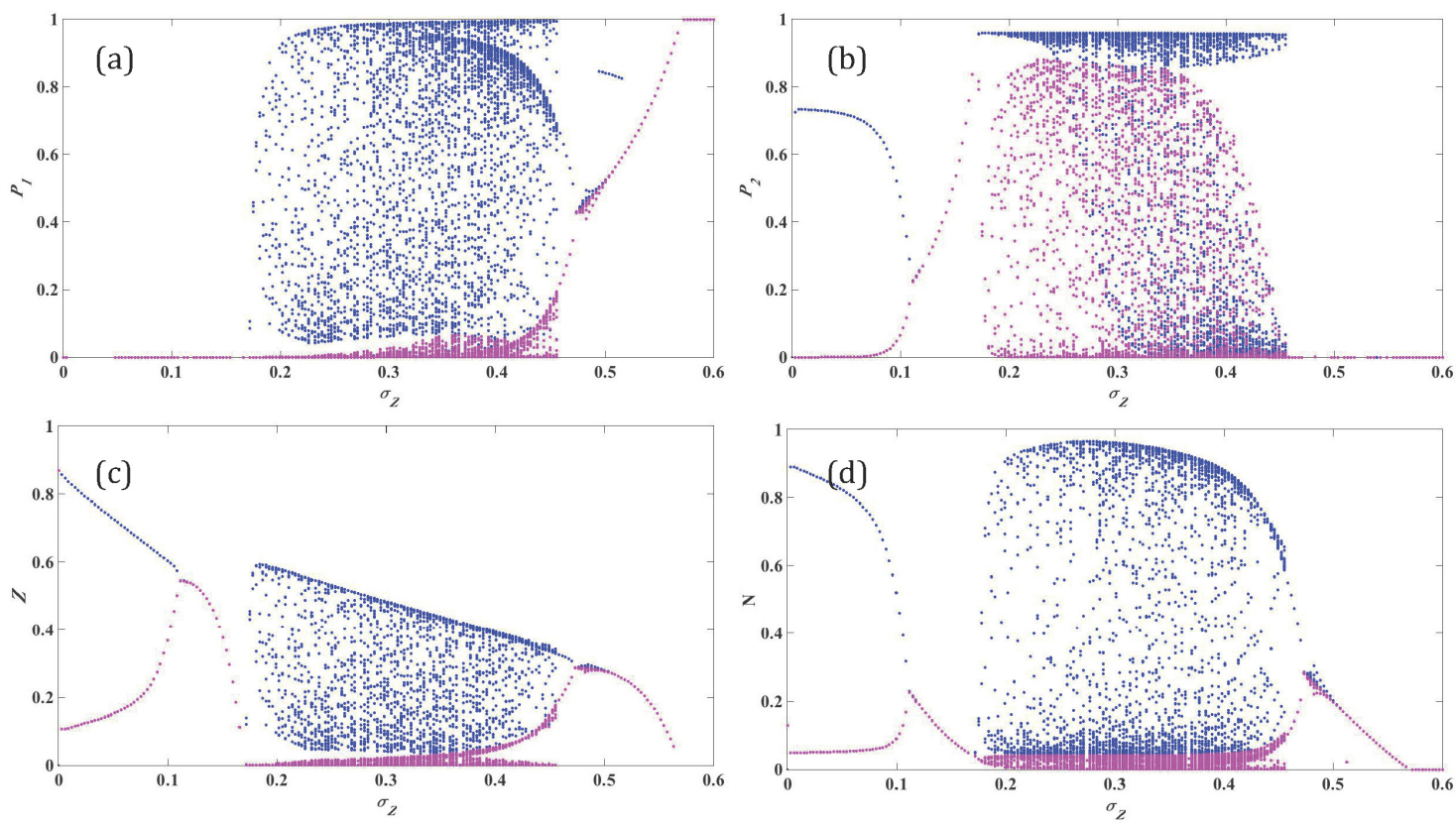

Figure 2. Bifurcation transition diagrams for the discriminate forager $(\rho=0)$ for $P_{1}(\mathbf{a}), P_{2}(\mathbf{b}), \boldsymbol{Z}(\mathbf{c})$, and $N$ (d) for values of the parameter $0<\sigma_{Z}<0.6$. Legend as Fig. 1 .

The difference in the dynamics of the indiscriminate and discriminate systems in the region $0.16<\sigma_{Z}<0.55$ is quite dramatic (cf Figs 1 and 2). The stable dynamics of the indiscriminate system, where $P_{1}$ and $P_{2}$ exchange mass in reducing sequential blooms while $\mathrm{Z}$ remains constant as the system approaches its equilibrium state are replaced by varying amplitude sequential blooms of $P_{1}$ then $P_{2}$ then $Z$ continue 
unabated. We found an extensive region of chaotic dynamics in the region $0.16<\sigma_{z}<0.55$ for the discriminate grazer (Fig 2) that was not evident for the indiscriminant grazer (Fig 1).
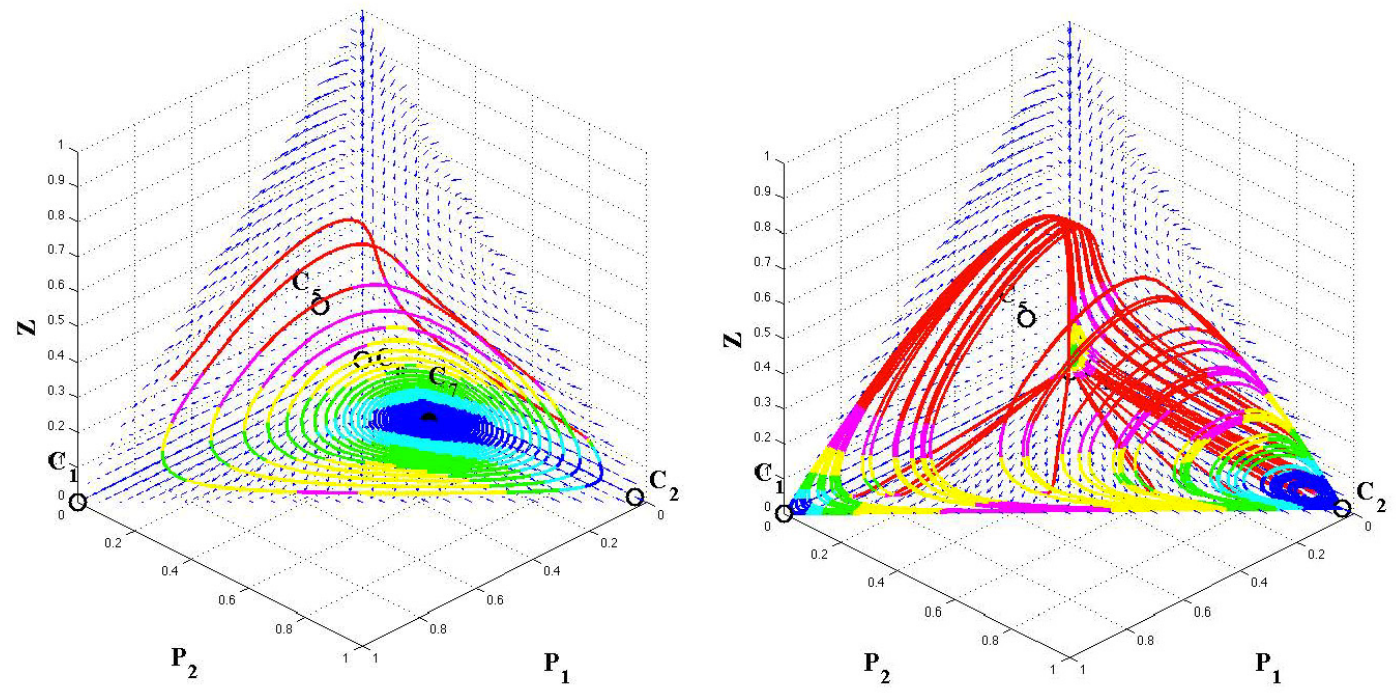

Figure 3. System dynamics for the indiscriminate forager ( $\rho=1$, left) and for the discriminate forager ( $\rho=0$, right) for $\sigma_{Z}=0.30$. Trajectories are coloured according to the non-dimensional velocity: blue

$=$ slow $\rightarrow$ red $=$ fast. Blue arrows show the vector field on the boundaries of the ecospace. Relevant critical points are shown as filled (stable) or unfilled (unstable). $\mathbf{C}_{1}$ is the $\left\{P_{1}^{*}, 0,0\right\}$ point, $\mathbf{C}_{2}$ is the

$$
\left\{0, P_{2}^{*}, 0\right\} \text { point, } \mathbf{C}_{5} \text { is the }\left\{P_{1}^{*}, Z^{*}, 0\right\} \text { point, and } \mathbf{C}_{7} \text { is the }\left\{P_{1}^{*}, P_{2}^{*}, Z^{*}\right\} \text { point. }
$$

The dynamics of the system in the chaotic region are very different with the dynamics of the indiscriminate grazer system rapidly contracting to a small region of the ecospace and then slowly approaching the equilibrium state, with most of the mass partitioned between $P_{1}$ and $P_{2}$ with small fractions in $Z$ and $N$ (Fig 3 , left panel). In contrast, the discriminate grazer system has states where $P_{1}$ dominates for an extended period (blue region in lower left corner of the right panel of Fig 3), states where $P_{2}$ dominates for an extended period (blue region in lower right corner of the right panel of Fig 3), and states where $Z$ briefly dominates in short-lived blooms (red trajectories in the right panel of Fig 3). These are fundamental differences that will lead the two models to produce very different predictions.

\section{DISCUSSION AND CONCLUSIONS}

Our results suggest that dynamic green ocean models (DGOMs), that are designed to simulate the carbon uptake in, and export from, the well-mixed upper ocean layers, should have carefully considered choices of grazing function. Indiscriminate grazing typically gives a model that can be readily, and stably, tuned to historical data. However, global warming can increase the closure terms, and thus increase the zooplankton mortality coefficient and move the system well into the obligate region. The changed outcomes in terms of which phytoplankton population dominates the ecology should be evaluated considering the use of the model:

- The primary producer least preferred by the grazer dominates the system initially;

- The balance between primary producer shifts as grazer mortality increases until the primary producer that leaves least nutrient for its competitor dominates the system;

- The transition between primary producer states is dependent on the grazing formulation - a simple transfer of biomass at steady state for indiscriminant grazing, but a chaotic transition for discriminant grazing.

This simple model suggests which parameter variations lead to desired modelling outcomes for ecosystem behaviour and raises the question of whether the plankton functional type responsible for controlling the carbon cycle in a model should be given properties more like $P_{1}$ or $P_{2}$. This study tries to clarify the influence of, and simplify those decisions about, the functional forms and the associated parameters to ensure stable, robust, desirable outcomes of the modelling process. The crucial choice of grazing strategies 
(indiscriminate/generalist versus discriminate/specialist) depends on whether the application outcome requires stable equilibrium populations or plankton successions.

\section{REFERENCES}

Anderson, T.R., Gentleman, W., Sinha, B., 2010. Influence of grazing formulations on the emergent properties of a complex ecosystem model in a global ocean general circulation model. Progress in Oceanography 87, 201-213.

Clarke, A., Tyler, P.A., 2008. Adult Antarctic Krill Feeding at Abyssal Depths. Current Biology 18, 282285.

Cropp, R.A., Moroz, I.M., Norbury, J., 2014. Chaotic dynamics in a simple dynamical green ocean plankton model. Journal of Marine Systems 139, 483-495.

Cropp, R.A., Norbury, J., 2009. Parameterizing plankton functional type models: insights from a dynamical systems perspective. Journal of Plankton Research 31, 939-963.

Cropp, R.A., Norbury, J., 2012a. Constructing Ecologies. Journal of Theoretical Biology 294, 1-8.

Cropp, R.A., Norbury, J., 2012b. The mechanisms of coexistence and competitive exclusion in complex plankton ecosystem models. Ecosystems 15, 200-212.

Cropp, R.A., Norbury, J., 2013. Modelling plankton ecosystems and the Library of Lotka. Journal of Marine Systems 125, 3-13.

Cropp, R.A., Norbury, J., 2015. Mixotrophy: the missing link in consumer-resource-based ecologies. Theoretical Ecology 8, 245-260.

Edwards, A.M., Brindley, J., 1996. Oscillatory behaviour in a three component plankton population model. Dyn. Stab. Sys. 11, 347-370.

Edwards, A.M., Brindley, J., 1999. Zooplankton mortality and the dynamical behaviour of plankton population models. Bulletin of Mathematical Biology 61, 303-339.

Edwards, A.M., Yool, A., 2000. The role of higher predation in plankton population models. Journal of Plankton Research 22, 1085-1112.

Fussmann, G.F., Heber, G., 2002. Foodweb complexity and chaotic population dynamics. Ecology Letters 5, 394-401.

Gentleman, W., Leising, A., Frost, B., Strom, S., Murray, J.D., 2003. Functional responses for zooplankton feeding on multiple resources: a review of assumptions and biological dynamics. Deep Sea Research Part II: Topical Studies in Oceanography 50, 2847-2875.

Holling, C.S., 1959. Some characteristics of simple types of predation and parasitism. Canadian Entomologist 91, 385-398.

Ivlev, V.S., 1961. Experimental ecology of the feeding of fishes. Yale University Press, New Haven.

Koen-Alonso, M., 2007. A process-oriented approach to the multi-species functional response. In: Rooney, N., McCann, K.S., Noakes, D.L.G. (Eds.), From Energetics to Ecosystems: The Dynamics and Structure of Ecological Systems. Springer, Netherlands, pp. 1-36.

Le Quéré, C., Harrison, S.P., Prentice, I.C., Buitenhuis, E.T., Aumonts, O., Bopp, L., Claustre, H., Cotrim da Cunha, L., Geider, R.J., Giraud, X., Klaas, C., Kohfeld, K.E., Legrende, L., Manizza, M., Platt, T., Rivkin, R.B., Sathyendranath, S., Uitz, J., Watson, A., Wolf-Gladrow, D., 2005. Ecosystem dynamics based on plankton functional types for global ocean biogeochemistry models. Global Change Biology 11, 2016-2040.

Loreau, M., 2010. From Populations to Ecosystems. Princeton University Press, Woodstock, Oxfordshire.

Lotka, A.J., 1925. Elements of physical biology. Wilkins and Kilkins, Baltimore.

Sailley, S.F., Vogt, M., Doney, S., Aita, M.N., Bopp, L., Buitenhuis, E.T., Hashioka, T., Lima, I., Le Quéré , C., Yamanaka, Y., 2013. Comparing food web structures and dynamics across a suite of global marine ecosystem models. Ecological Modelling 261-262, 43-57.

Steele, J.H., Henderson, E.W., 1992. The role of predation in plankton models. Journal of Plankton Research $14,157-172$.

Vallina, S.M., Ward, B.A., Dutkiewicz, S., Follows, M., 2014. Maximal feeding with active prey-switching: a kill-the-winner functional response and its effects on global diversity and biogeography. Progress in Oceanography 120, 93-109.

Visser, A.W., Fiksen, O., 2013. Optimal foraging in marine ecosystem models: selectivity, profitability and switching. Marine Ecology in Progress Series 473, 91-101.

Volterra, V., 1926. Variations and fluctuations of the numbers of individuals in animal species living together. In: Reprinted in 1931 in Chapman, R.N. (Ed.), Animal Ecology. McGraw Hill, New York. 